\title{
NOTE ON POSITIVE LINEAR OPERATORS
}

\section{GARRETT BIRKHOFF}

Let $\mathscr{L}$ be a topological linear space, and let $\mathfrak{C}$ be a closed convex cone in $\mathfrak{L}$ such that $\mathfrak{L}=\mathfrak{C}-\mathfrak{C}$, i.e., such that $\mathfrak{L}=(\mathfrak{L}, \mathfrak{C})$ is a directed topological linear space. ${ }^{1}$

LEMMA 1. If $\gamma_{n} \rightarrow \gamma, f_{n} \rightarrow f, g_{n} \rightarrow g$, and $\gamma_{n} f_{n} \geqq g_{n}$ for $n=1,2,3, \cdots$, then $\gamma f \geqq g$.

For, $\gamma f-g=\lim \left(\gamma_{n} \hat{f}_{n}-g_{n}\right) \in \mathcal{C}$ since $\mathfrak{C}$ is closed.

Corollary. $\mathscr{L}=(\mathfrak{L}, \mathfrak{e})$ is an Archimedean directed vector space.

For, if $0 \leqq g \leqq \gamma_{n} f$ where $\gamma_{n} \downarrow 0$, then $g \leqq 0 f=0$.

Conversely, any Archimedean directed vector space $\mathscr{L}=(\mathscr{L}, \mathfrak{e})$, given its intrinsic order or relative uniform topology, is a topological linear space in which $\mathfrak{C}$ is a closed convex cone with $\mathfrak{L}=\mathfrak{C}-\mathfrak{C}$. Hence our results apply to vector lattices in their usual intrinsic topologies.

Now let $\theta(f, g)$ be the projective quasi-metric on $\mathbf{e}-0$ defined by

$$
\begin{gathered}
\theta(f, g)=\ln \left(\alpha_{0} \beta_{0}\right), \text { where } \\
\alpha_{0}=\inf \{\alpha \mid \alpha f \geqq g\}, \quad \beta_{0}=\inf \{\beta \mid \beta g \geqq f\} .
\end{gathered}
$$

Lemma 2. The projective quasi-metric $\theta(f, g)$ is a lower-semicontinuous function on $\mathrm{e} \times \mathfrak{e}$.

Proof. Let. $f_{n} \rightarrow f$ and $g_{n} \rightarrow g$ in $\mathcal{E}$, and let $\alpha_{n}$ and $\beta_{n}$ be the least numbers such that $\alpha_{n} f_{n} \geqq g_{n}$ and $\beta_{n} g_{n} \geqq f_{n}$. These exist by Lemma 1 and are positive since $\mathcal{L}$ is Archimedean, and $\theta\left(f_{n}, g_{n}\right)=\theta_{n}$ satisfies $e^{\theta_{n}}$ $=\alpha_{n} \beta_{n}$. Let $\theta=\lim \inf \theta_{n}$. The case $\theta=\infty$ is trivial, since it imposes no restriction on $\theta(f, g)$. Moreover, by restricting attention to a subsequence, we can reduce to the case $\theta=\lim \theta_{n}$.

This may increase the values of $\alpha=\lim \inf \alpha_{n}$ and $\beta=\lim \inf \beta_{n}$. But both $\alpha>0$ and $\beta>0$ since, by Lemma 1 , $\alpha$ f $\geqq g>0$ and $\beta g \geqq f$ where $\alpha \beta \leqq e^{\theta}$ and $\mathcal{L}$ is Archimedean. It follows that $\alpha<+\infty$ and $\beta<+\infty$. Now extract a subsequence $\alpha_{n} \rightarrow \mathbf{\alpha}$; it will follow that $\beta_{n}=e^{\theta_{n}} / \alpha_{n} \rightarrow e^{\theta} / \mathbf{\alpha} \geqq \boldsymbol{\beta}$. Moreover, by Lemma 1 , af $\geqq g$ and $(e \theta / \mathbf{\alpha}) g \geqq f$, whence

$$
\theta(f, g) \leqq \ln \left[\mathbf{a}\left(e^{\boldsymbol{\theta}} / \mathbf{a}\right)\right]=\boldsymbol{\theta}=\lim \inf \theta\left(f_{n}, \mathbf{g}_{n}\right) .
$$

This proves the lemma.

Received by the editors April 30, 1963 and, in revised form, August 1, 1963.

${ }^{1}$ For the terminology used here, and the background of the present discussion, see $[1]$. 
COROLlaRy 1. For any continuous strictly positive linear operator $P$ on $(\mathcal{L}, \mathfrak{C})$, the function $\delta(f)=\theta(f, f P)$ is lower-semicontinuous on $\mathfrak{C}$.

Note that, in the (intrinsic) relative uniform topology on any Archimedean directed vector space, any positive linear operator is continuous [1, Lemma 2].

THEOREM 1. Let $P$ be any completely continuous strictly positive linear operator on the partially ordered ${ }^{2}$ Banach space $\mathfrak{L}=(\mathfrak{L}, \mathfrak{C})$, and let $\delta_{0}=\inf _{\mathfrak{C}} \delta(f)$. Then the set of nonzero $f \in \mathcal{C}$ where $\delta(f)=\delta_{0}$ is a nonvoid closed cone $\Delta_{0}$, invariant under $P$.

Proof. Let $S$ be the unit sphere. Since $\delta(\lambda f)=\delta(f)$ for any positive scalar $\lambda, \delta_{0}=\inf _{S \cap \mathcal{C}} \delta(f)$. Moreover, since $\theta\left(f P, f P^{2}\right) \leqq \theta(f, f P)$ by $[1,(11)], \delta(f P) \leqq \delta(f)$ and so $\delta_{0}=\inf _{S P \cap \mathcal{e}} \delta(f)$. But by hypothesis, $S P \cap \mathbb{e}$ has a compact closure $\mathcal{K}$. Hence every sequence of $f_{n} \in S \cap \mathfrak{e}$ with $\delta\left(f_{n}\right)=\theta\left(f_{n}, f_{n} P\right) \downarrow \delta_{0}$ has a limit point $g \in \mathcal{K}$, where $g \in \mathfrak{C}$ since $\mathfrak{e}$ is closed, and so some $\alpha g \in S \cap \mathfrak{e}, \alpha>0$. Further, by Lemma 2:

$$
\theta(\alpha g, \alpha g P) \leqq \theta(g, g P) \leqq \lim \inf \theta\left(f_{n} P, f_{n} P^{2}\right) \leqq \lim \inf \theta\left(f_{n}, f_{n} P\right)=\delta_{0}
$$

since $\theta(h P, k P) \leqq \theta(h, k)$ for any $h, k \in \mathcal{C}$. Consequently $\delta(f)$ assumes the values $\delta_{0}$ on a nonvoid closed set $\Delta_{0}$. This set is a cone invariant under $P$ since, as noted above, $\delta(f P) \leqq \delta(f)$ and $\delta(\lambda f)=\delta(f)$.

Obviously, the Theorem of Jentzsch refers to the special case $\delta_{0}=0$. In this case, $\Delta_{0}$ is the cone of invariant directions, and the preceding argument is closely related to proofs of Jentzsch's Theorem by Kreln and Rutman.

It would be interesting to extend Theorem 1 to Archimedean directed vector spaces which are not Banach spaces. It would be even more interesting to know more about the structure of the set $\Delta_{0}$. In this connection, the following example is relevant.

EXAMPLE 1. Let $\&$ be the vector lattice of all continuous functions on $[0,1]$, let $\mathfrak{C}$ consist of all nonnegative $f(x)$, and let $P[f(x)]$ $=p(x) f(x)$, where $p(x)$ is a positive continuous function.

One easily verifies that, in Example 1, $P$ is an isometry for the projective quasi-metric:

$$
\theta(f, g)=\theta(f P, g P) \quad \text { for all } f, g \in \mathbb{C} .
$$

Moreover

$$
\theta(f, f P)=m=\ln [\sup p(x)] /[\inf p(x)]
$$

2 We continue to assume that $\mathcal{C}$ is closed and that $\mathscr{L}=\mathfrak{C}-\mathbb{C}$. 
is independent of $f$ : all elements of $\mathfrak{e}$ are moved through the same distance. The only transformations of Euclidean space which satisfy (3)-(4) are translations, but the analogy is very poor: the projective quasi-metric defined by $(1)-\left(1^{\prime}\right)$ has little in common with Euclidean distance.

Unfortunately, Example 1 (in which $\Delta_{0}=\mathfrak{e}$ ) does not seem to be typical. Not only is $\mathbb{e} P$ not compact, but in general the set $\Delta_{0}$ is not even convex, on the connected components of $\mathfrak{e}$. This is shown by the following example constructed by Mr. Alan G. Waterman. ${ }^{3}$

Example 2 (Waterman). Let $P$ be the linear operator

(5) $(x, y, u, v) \rightarrow(x,(x+v) /(k+1), v /(k-1)+(k-2) u /(k-1), v)$.

Let $f=(1,1,1, k)$ and $g=(k, 2,1,1)$. Then, for any $k>2$, we have $\delta(f)=\delta(g)=\ln 2$, but

$$
\delta(f+g)=\ln (9 / 4)>\max \{\delta(f), \delta(g)\} .
$$

The author wishes to thank Professor Hugh Gordon and the referee for helpful criticisms and suggestions.

\section{REFERENCES}

1. G. Birkhoff, Uniformly semi-primitive multiplicative processes, Trans. Amer. Math. Soc. 104 (1962), 37-51.

2. M. G. Kreln and M. A. Rutman, Linear operators leaving invariant a cone in a Banach space, Uspehi Mat. Nauk 3 (1948), 3-95; Amer. Math. Soc. Transl. No. 26.

\section{HARVARD UNIVERSITY}

'Mr. Waterman's work was supported by the National Science Foundation under Grant GP 595. 